\title{
Does the incorporation of strontium into calcium phosphate improve bone repair? A meta-analysis
}

Ming-Dong Yan ${ }^{1 \dagger}$, Yan-Jing Ou ${ }^{1,2+}$, Yan-Jun Lin ${ }^{1 \dagger}$, Rui-Min Liư ${ }^{3}$, Yan Fang ${ }^{4}$, Wei-Liang Wu' ${ }^{1}$ Lin Zhou', Xiu Yao ${ }^{1,5}$ and Jiang Chen ${ }^{1 *}$

\begin{abstract}
Background: The application of calcium phosphate (CaP)-based bone substitutes plays an important role in periodontal regeneration, implant dentistry and alveolar bone reconstruction. The incorporation of strontium (Sr) into CaP-based bone substitutes appears to improve their biological properties, but the reported in vivo bone repair performance is inconsistent among studies. Herein, we conducted a systematic review and meta-analysis to investigate the in vivo performance of Sr-doped materials.

Methods: We searched PubMed, EMBASE (via OVIDSP), and reference lists to identify relevant animal studies. The search, study selection, and data extraction were performed independently by two investigators. Meta-analyses and sub-group analyses were conducted using Revman version 5.4.1. The heterogeneity between studies were assessed by $l^{2}$. Publication bias was investigated through a funnel plot.

Results: Thirty-five studies were finally enrolled, of which 16 articles that reported on new bone formation (NBF) were included in the meta-analysis, covering 31 comparisons and 445 defects. The overall effect for NBF was 2.25 (95\% $\mathrm{Cl} 1.61-2.90, \mathrm{p}<0.00001, \mathrm{I}^{2}=80 \%$ ). Eight comparisons from 6 studies reported the outcomes of bone volume/tissue volume (BV/TV), with an overall effect of $1.42\left(95 \% \mathrm{Cl} 0.65-2.18, \mathrm{p}=0.0003, \mathrm{I}^{2}=75 \%\right)$. Fourteen comparisons reported on the material remaining (RM), with the overall effect being $-2.26\left(95 \% \mathrm{Cl}-4.02\right.$ to $\left.-0.50, p=0.0009, \mathrm{I}^{2}=86 \%\right)$.

Conclusions: Our study revealed that Sr-doped calcium phosphate bone substitutes improved in vivo performance of bone repair. However, more studies are also recommended to further verify this conclusion.
\end{abstract}

Keywords: Bone defects, Calcium phosphate, Strontium, Bone regeneration, Meta-analysis

*Correspondence: jiangchen@fimu.edu.cn

${ }^{\dagger}$ Ming-Dong Yan, Yan-Jing Ou and Yan-Jun Lin contributed equally to this study

${ }^{1}$ Fujian Key Laboratory of Oral Diseases and Fujian Provincial Engineering Research Center of Oral Biomaterial and Stomatological Key Laboratory of Fujian College and University, School and Hospital of Stomatology, Fujian Medical University, Fuzhou 350002, China

Full list of author information is available at the end of the article

\section{Introduction}

At present, the repair of alveolar bone defects caused by disease, trauma, periodontitis, or congenital malformation is facing challenge, especially for patients with large bone defects or systemic diseases (such as osteoporosis or diabetes) [1, 2]. Although autologous bone grafting is considered to be the gold standard, its clinical applicability is limited owing to the need to open up a second surgical area and possible complications at the donor site [3, 4]. 
Nowadays, calcium phosphate $(\mathrm{CaP})$ ceramics - as a representative material for synthetic bone substituteshave been widely used in periodontal regeneration and alveolar bone reconstruction $[4,5]$. However, traditional calcium phosphate materials have insufficient osteogenic ability and degradation performance. To improve the biological properties of these bone substitutes, researchers have attempted to incorporate bioinorganic ions into CaP-based materials [6].

Among various bioinorganic ions, strontium $(\mathrm{Sr})$ has attracted significant research attention in the past ten years [7]. Sr is known to be a trace element in the human body and plays an important role in bone metabolism $[8$, 9]. It is conducive to osteogenesis, and can be mixed with hydroxyapatite (HA) through surface exchange or ion substitution, leading to the increase of bone mineral content and bone density, which improves bone regeneration and repair.

Several studies have investigated the effects of the addition of Sr on the physicochemical properties and in vitro/ in vivo behaviour of CaP-based bone substitutes. Tao et al. demonstrated that the calcium phosphate doped with $\mathrm{Sr}$ has a faster absorption rate [10]. In addition, Srsubstituted biomaterials increased the differentiation of osteoblasts and activated the expression of pro-osteogenic molecules used for bone remodelling [11-13]. A number of in vivo studies have shown that Sr-enhanced calcium phosphate materials have better osteogenic properties in vivo [14-16]. However, other study found that no positive effect was observed in terms of promoting in vivo bone regeneration [17].

In view of the differences among studies regarding the effects of Sr-doped CaP-based materials, it becomes imperative to conduct a systematic review and metaanalysis. In addition, sub-group analyses based on different animals, material types, and implantation periods were also conducted. The main purpose of this study was to systematically review the synthesis method and characteristics - such as crystallinity, particle size, and porosity-of included Sr-doped ( $\mathrm{CaP}$ ) materials and to analyse the properties of new bone formation (NBF) and material degradation in vivo.

\section{Methods}

\section{Search strategy}

The methodology of this study followed the recommendations of the Systematic Review Centre for Laboratory Animal Experimentation (SYRCLE) guidelines [18] and the guidelines of the PRISMA statement (http://www. prisma-statement.org/). In vivo studies that evaluate the effects of Sr-doped (CaP)-based materials from database inception to December 2020, without any language restrictions, were identified by searching the PubMed and
EMBASE (via OVIDSP) databases. This paper combined the MeSH heading and text search strategies, with multiple terms associated with 'bone regeneration', 'strontium, 'bone substitutes', and 'animal research' were used. Since tricalcium phosphate (TCP), HA, anhydrous dicalcium phosphate (TTCP), and tetracalcium phosphate (DCPA) are commonly used materials in this field of research, these terms have been also used as search words in the search formula. Search filters were utilized to detect all publications concerning animal studies $[19,20]$. The detailed search strategies for PubMed and Embase are shown in Additional file 1: Table S1 and Additional file 2: Table S2, respectively. In addition, we manually searched the reference lists of major research journals and review papers to identify additional relevant studies.

\section{Eligibility criteria}

Two investigators (Y-M.D. and L-R.M.) independently screened potentially eligible studies. Any disagreement was resolved by discussion and consensus among reviewing authors. The inclusion criteria were (1) original animal studies on bone defects, (2) comparisons of Sr-doped and $\mathrm{Sr}$-free $(\mathrm{CaP})$-based bone substitutes; and (3) outcomes of histological, micro-CT, or histomorphometric data.

\section{Study selection and data extraction}

Two authors independently reviewed studies considered for inclusion in the meta-analysis and performed data extraction. We used an existing data extraction method to retrieve data regarding the basic characteristics. For all included papers, the outcome data for the experimental and control groups were extracted if the mean, standard deviation (SD) or standard error (SE), and the number of defects per group $(\mathrm{N})$ were reported or could be recalculated. If the data were presented only in graphical form, pictures were converted to data using the WebPlotDigitizer tool (available online at https://automeris.io/WebPl otDigitizer/), which was considered to have high levels of intercoder reliability and validity [21].

\section{Quality assessment}

The risk-of-bias assessment was based on SYRCLE's Risk of Bias (RoB) tool, which is specifically designed for animal studies. Two authors independently assessed the risk of bias.

\section{Statistical analysis}

The primary outcome of interest was the pooled overall NBF. Bone volume/tissue volume (BV/TV) and remaining material (RM) were the second outcomes of interest. Quantitative meta-analysis was performed when more than one study presented relevant data. Standardized 


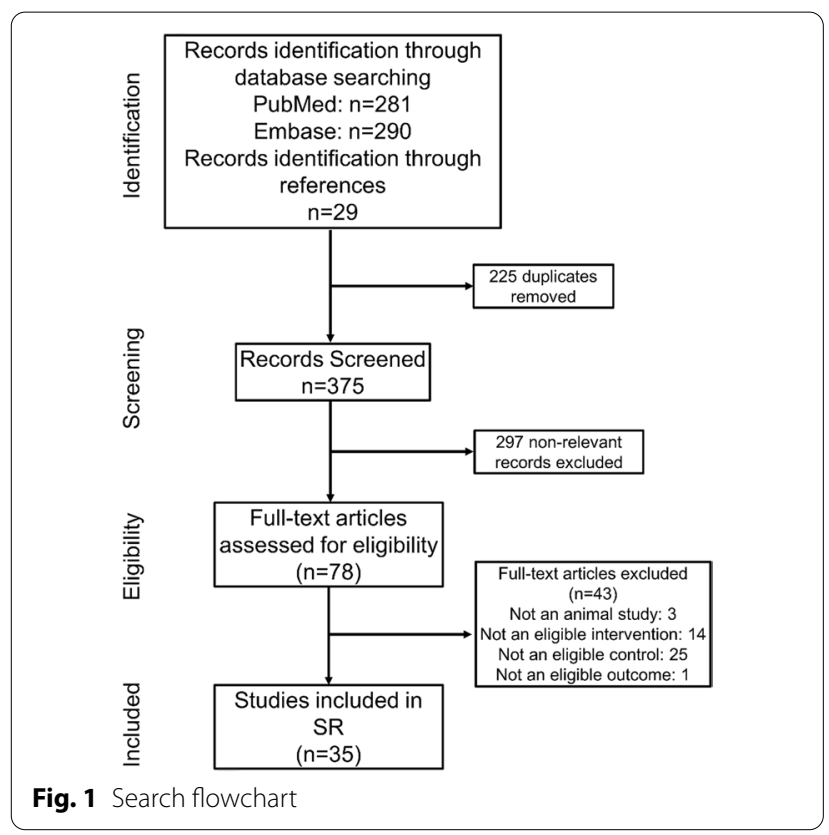

mean differences (SMD) or mean differences (MD) and 95\% confidence interval $(95 \% \mathrm{CI})$ were calculated. Heterogeneity was assessed using $\mathrm{I}^{2}$. An $\mathrm{I}^{2}$ value greater than $50 \%$ was considered to indicate significant heterogeneity. However, because of the underlying methodological heterogeneity (e.g. baseline characteristics of the animals, sample sizes, and implantation periods), we used the DerSimonian and Laird random-effects model for all analyses. Potential sources of between-study heterogeneity were explored by subgroup analyses according to the following factors, whenever appropriate: physical condition (health vs disease); animals (e.g. rat, rabbit, or sheep); materials; and implantation periods. We reported p-values for each covariate. Publication bias was investigated through a funnel plot. Analyses were conducted using Review Manager (version 5.4.1, The Cochrane Collaboration, 2020).

\section{Results}

\section{Paper identification and selection}

Through the search, a total of 600 related articles were retrieved, including 281 from Pubmed, 290 from Embase, and 29 from reference lists. After removing duplicates and screening all titles and abstracts, 78 potential studies were selected for full-text evaluation. Finally, 35 papers $[10,11,13-17,22-49]$ met the inclusion criteria and were included in the systematic review (Fig. 1).

\section{Characteristics of included studies}

The 35 included studies were published from 2001 to 2020, and the curve of the cumulative number of papers included in the systematic review each year is shown in Fig. 2, indicating the increasing amount of attention this topic has received in the past decade.

Among these studies, one used a dog model [48], one used a mouse model [46], three used sheep models $[26,39,41]$, thirteen used rabbit models $[11,13,15,28$, $30,31,33-35,37,38,45,47]$, and seventeen used rat

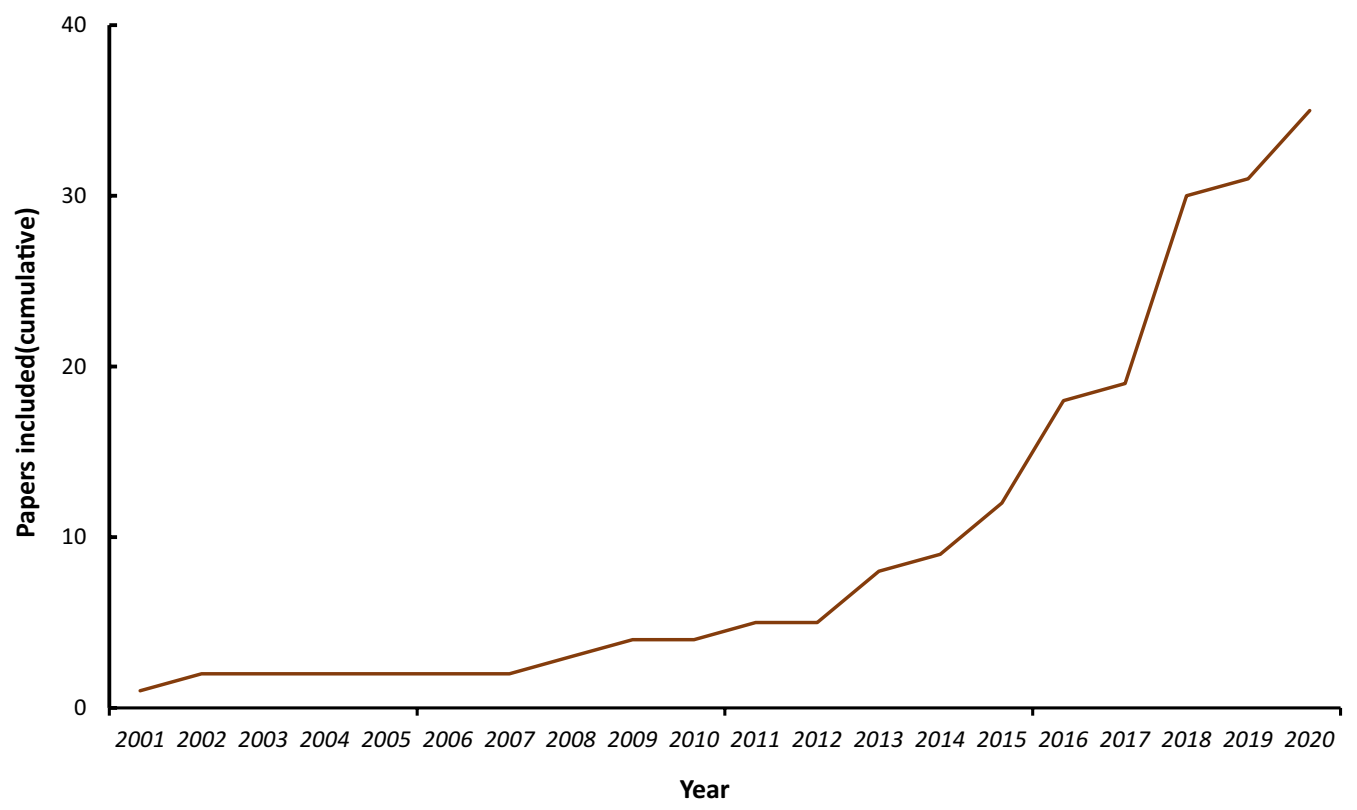

Fig. 2 Cumulative number of papers included in the systematic review each year 
models $[10,14,16,17,22-25,27,29,32,36,40,42-44$, 49]. There were nineteen studies focused on healthy animals $[15,23,28-31,33,35-41,45-49]$, one on albinism [24], one on osteonecrosis [34], and thirteen on osteoporosis [10,11, 14, 16, 17, 22, 25-27, 32, 42-44]. The main characteristics of the included studies are listed in Table 1.

Various forms of biomaterials were reported in these studies, including cylindrical, granular/powder, spheri$\mathrm{cal}$, and disc-shaped. The sites of the bone defects were widely distributed, including the vertebra in one study, humerus in one study, femur and tibia in one study, mandible in two studies, maxilla in two studies, radius in three studies, tibia in three studies, calvaria in four studies, and femur in 18 studies.

\section{Biomaterial characteristics}

The included studies contained multiple types of calcium phosphate materials. Different synthesis methods, crystallinities, particle sizes, implant morphologies, porosities, stoichiometries, and thermal treatments could influence the biological properties and in vivo efficacy of these materials. Table 2 summarizes the characteristics of all materials used in the included research.

\section{Risk of bias and quality assessment}

The risk of bias of the included studies was relatively high (Fig. 3A). Among them, only one paper [29] provided a sufficient and reasonable description of the generation of random sequences. Furthermore, it was difficult to confirm the accurate baseline characteristics in each group as none of the studies offered complete baseline information. None of the papers reported on the 'allocation concealment' and 'blinding of performance bias.' 'Random housing' was considered as a 'low risk of bias' in six publications [10, 24, 33, 42, 43, 46] (17\%), and only five of the articles [30, 31, 34, 37, 48] (14\%) reported 'random selection for outcome assessment'. Eight articles [15, 23, 26, $34,39,44,47,48$ ] $(23 \%)$ were considered to have a 'low risk of bias' in terms of the 'blinded outcome reviewers', while two papers $[22,44]$ were considered to have a 'high risk of bias' in terms of 'incomplete data reporting. Moreover, in terms of 'selective outcome reporting' and 'other sources of bias', a majority of the articles were defined as having a 'low risk of bias'.

Another three quality indicators for the 35 studies are presented in Fig. 3B. For 17 studies (less than 50\%), it was reported that the experimental groups were randomized in some way. Less than $1 / 4$ of the studies reported 'blinding of the experiment', and only two articles $[23,48]$ mentioned the 'power/sample size calculation'.

\section{Meta-analysis of new bone formation from histological outcomes}

A total of 18 articles were included in our meta-analysis, covering 31 comparisons and 445 defects. In this analysis, the pooled effect for NBF was 2.25 (95\% CI 1.61-2.90), indicating a significantly higher NBF for Sr-doped materials (Fig. 4).

Table 3 shows the outcomes of the subgroup analysis for NBF. In both the healthy animal group and osteoporosis models, the $\mathrm{Sr}$-enriched material significantly increased NBF, with (SMD: $1.85[0.95,2.76], \mathrm{I}^{2}=81 \%$ ) and (SMD: $2.73[1.94,3.52], \mathrm{I}^{2}=71 \%$ ), respectively. According to the included studies, a superior bone repairing effect could be found in healthy animals. A forest plot of this is provided in Additional file 3: Fig. S1. For animal models, one rat study [23], one rabbit study [47], and one sheep study [39] reported lower NBF in the Srdoped group without statistical significance during short implantation periods (1 week-30 days). Results of other studies and meta-analyses all suggested that $\mathrm{Sr}$ doping could significantly promote NBF (Additional file 3: Fig. S2). Sub-group analyses of different calcium phosphates (HA, $\beta$-TCP, CPC, and CPP) and different follow-up periods ( 1 month, 2 months, 3 months, and 4 months) both supported the conclusion that $\mathrm{Sr}$-doping enhanced NBF. However, high heterogeneity could be observed in all subgroups, with $\mathrm{I}^{2}$ values ranging from 52 to $87 \%$ (Additional file 3: Fig. S3 and Additional file 3: Fig. S4).

\section{Meta-analysis of new bone formation from micro-CT assessment}

Micro-CT measurements of bone volume/tissue volume (BV/TV) were performed in five of the articles included in the meta-analysis. The overall effect of BV/ TV was $1.42(95 \%$ CI $0.65-2.18, \mathrm{p}<0.05)$, suggesting that $\mathrm{Sr}$ enrichment promoted NBF and bone regeneration (Fig. 5).

\section{Meta-analysis of the remaining materials}

In terms of material absorption, the histological outcomes were extracted from six articles, among which four comparisons found that material remained for less than 1 month, six comparisons between 1 and 3 months, and four comparisons for more than 3 months. The results showed that, in the early stages ( $\leq 1$ month), the absorption of the Sr-doped material was less than that of the non-Sr-doped group $(3.11[-0.38,6.60])$. In the middle (1-3 months) and longer ( $>3$ months) periods, the absorption of the Sr-doped material was significantly higher than that of the Sr-free group (Fig. 6).

Subgroup analysis was also conducted for different material types (HA, $\beta$-TCP, CPC, and CPP). The results 


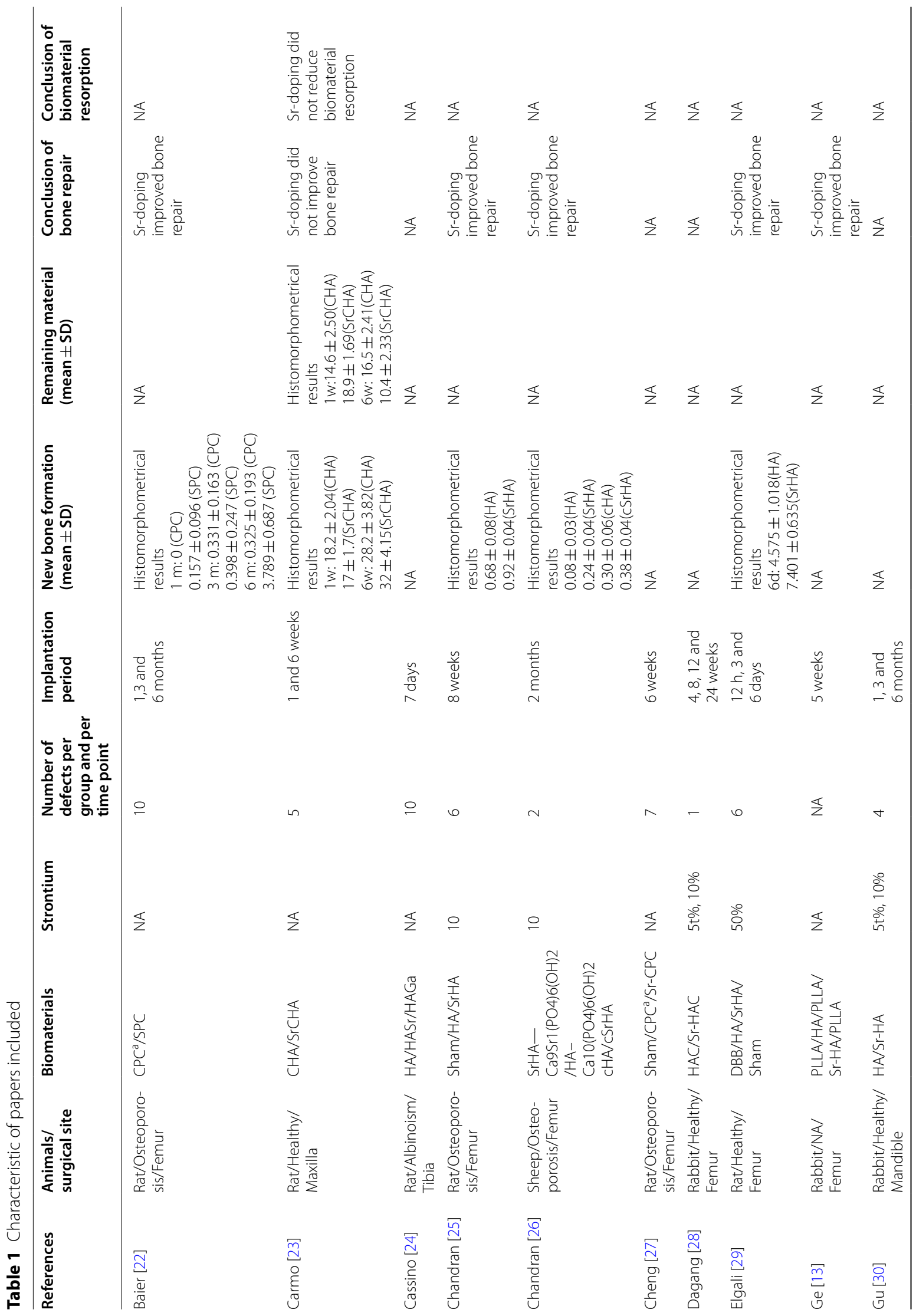




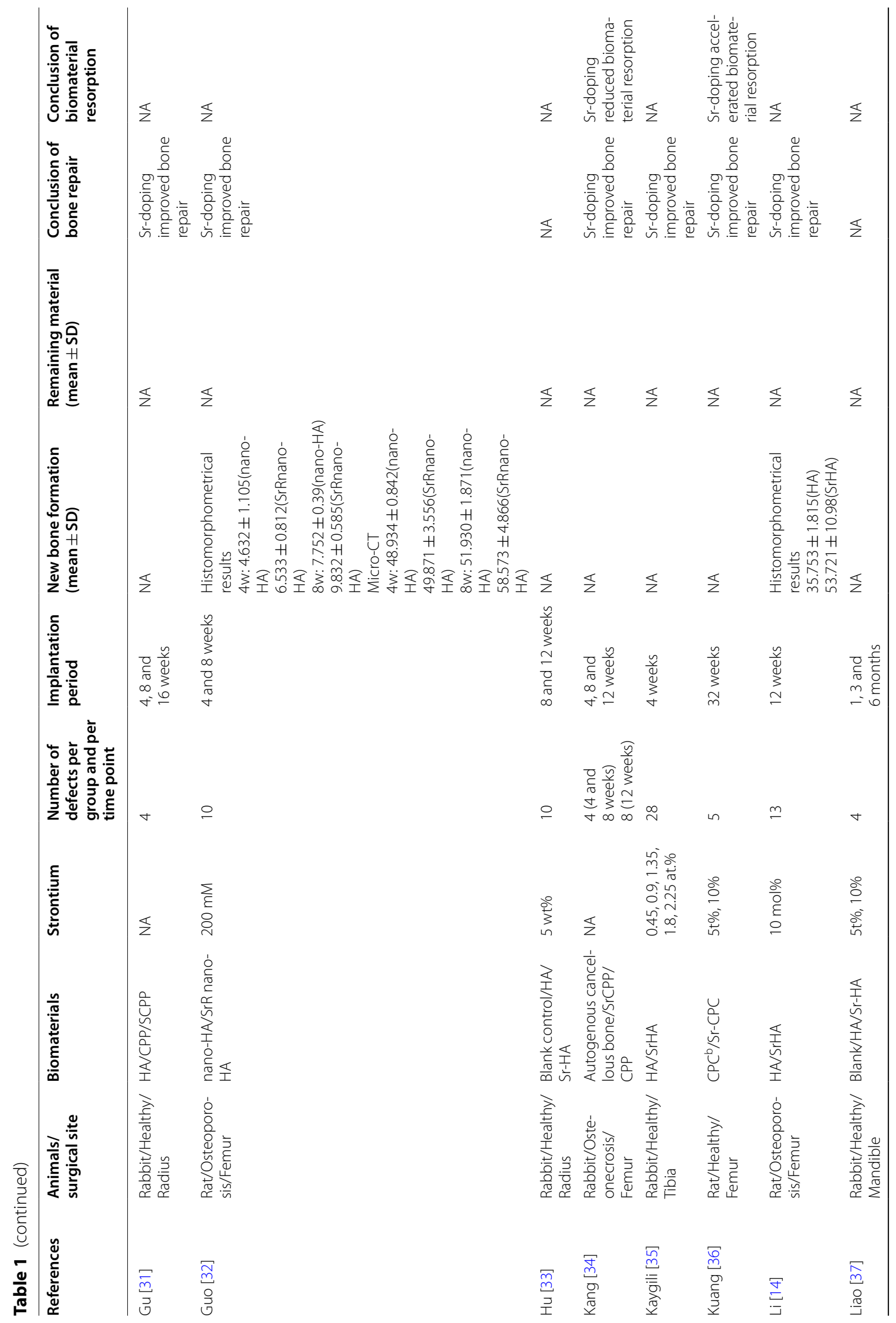




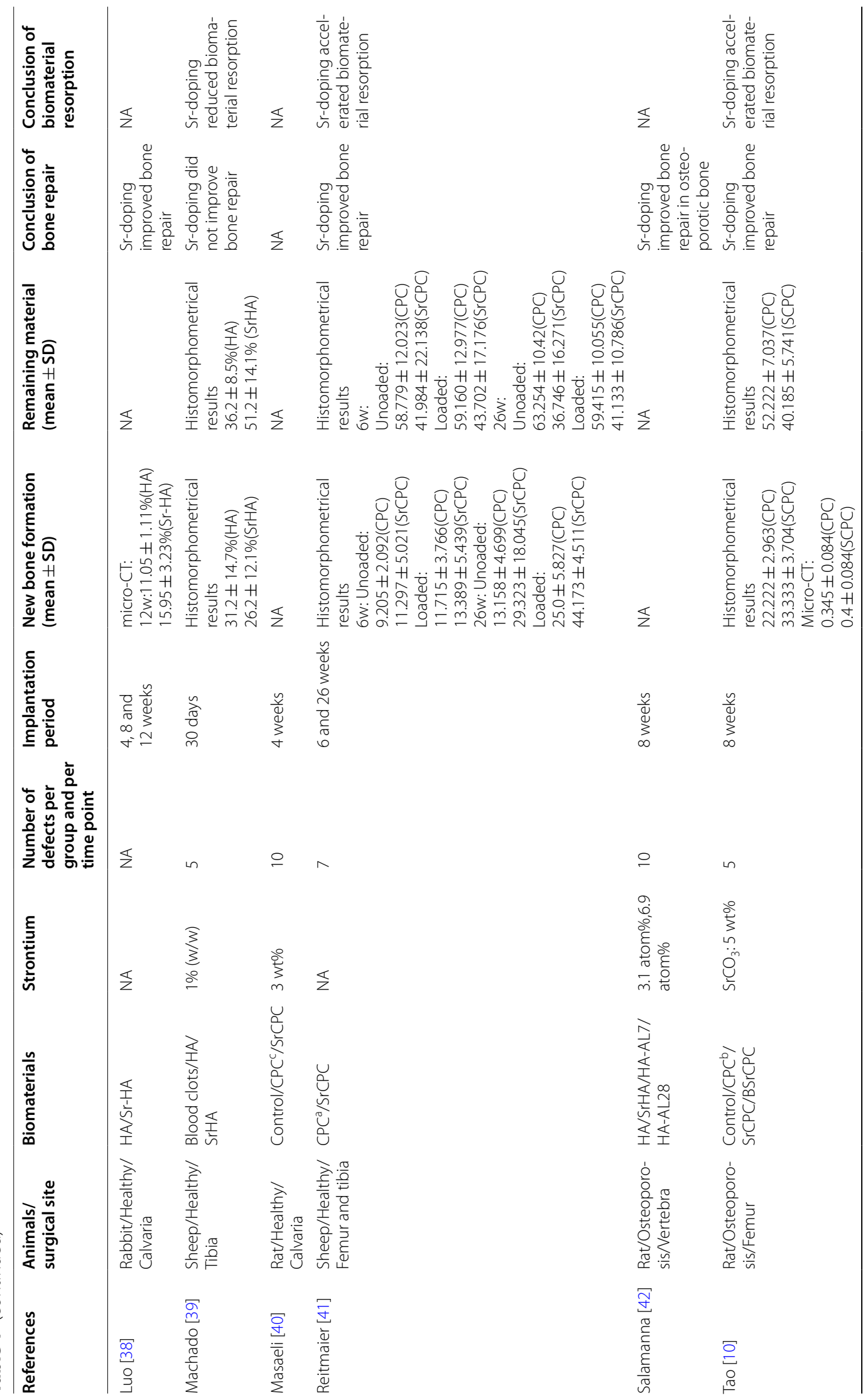




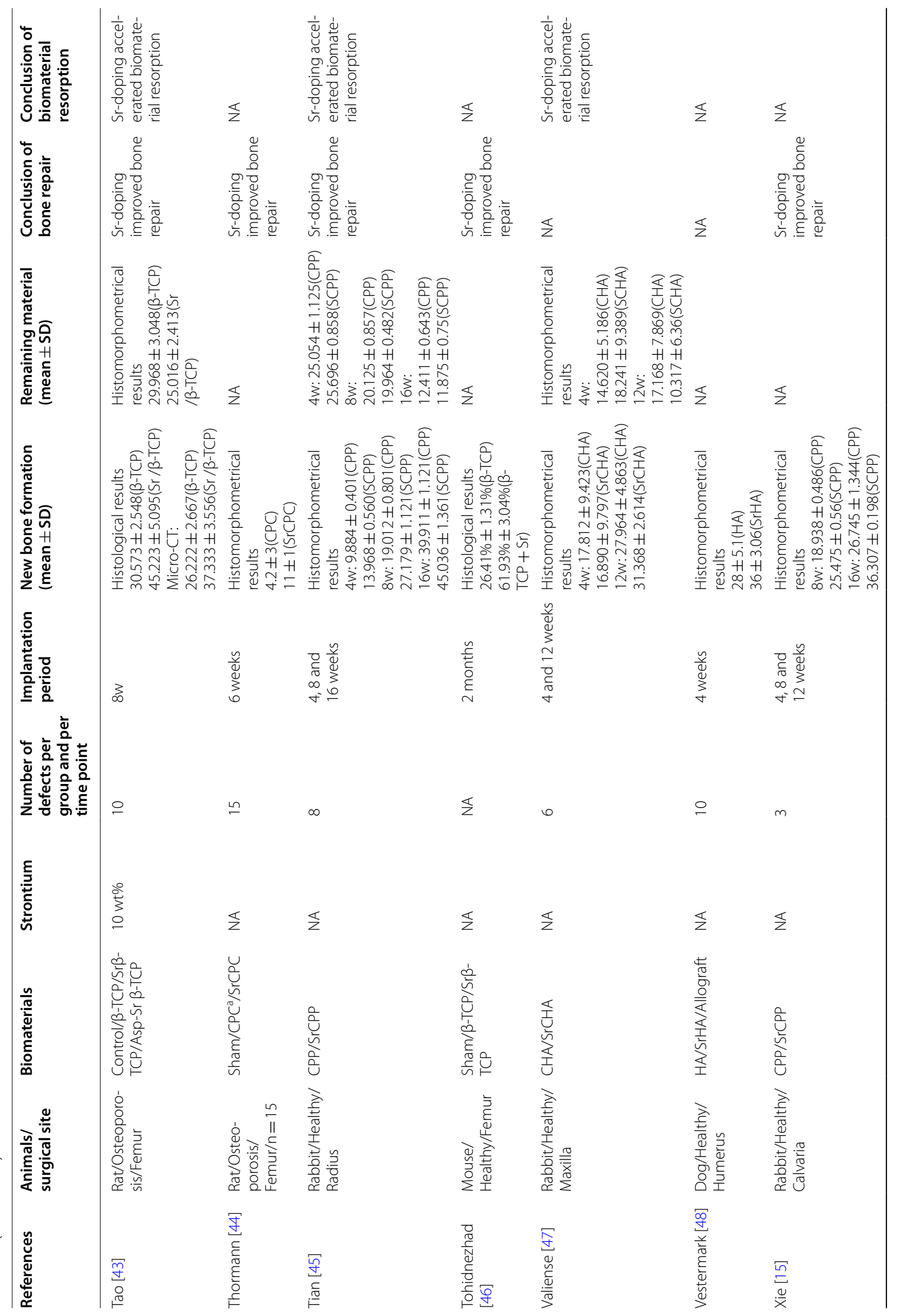




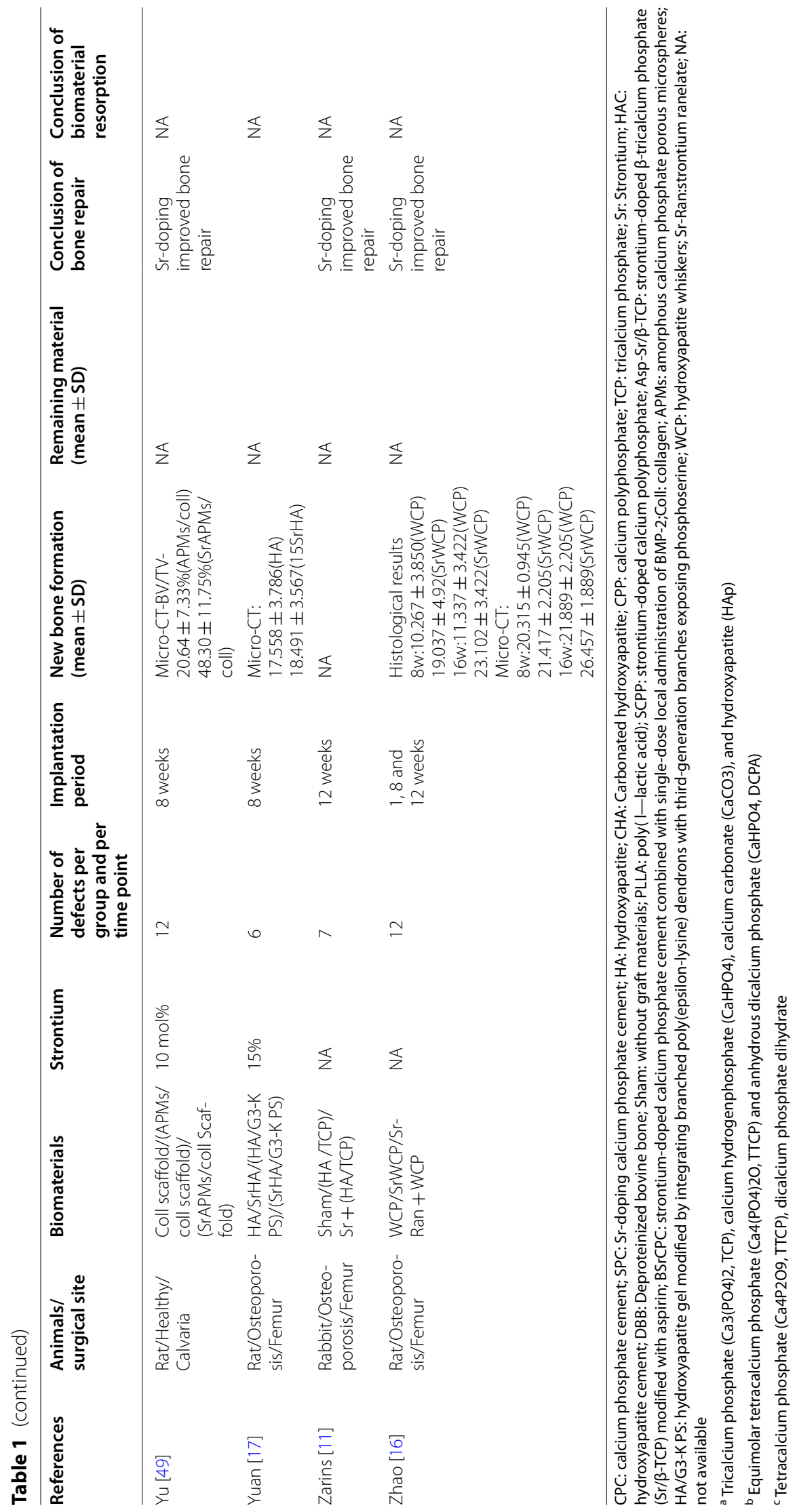




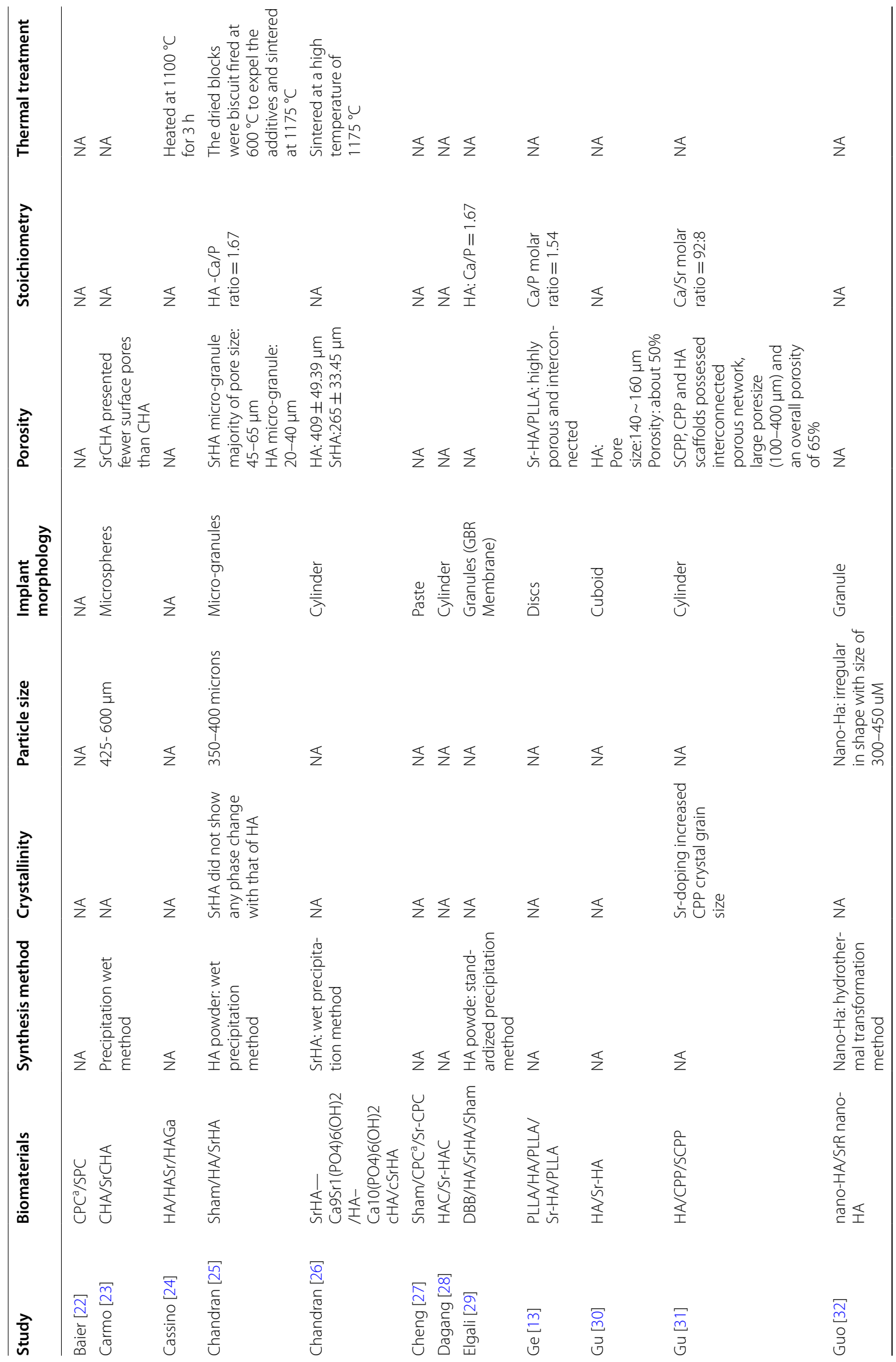




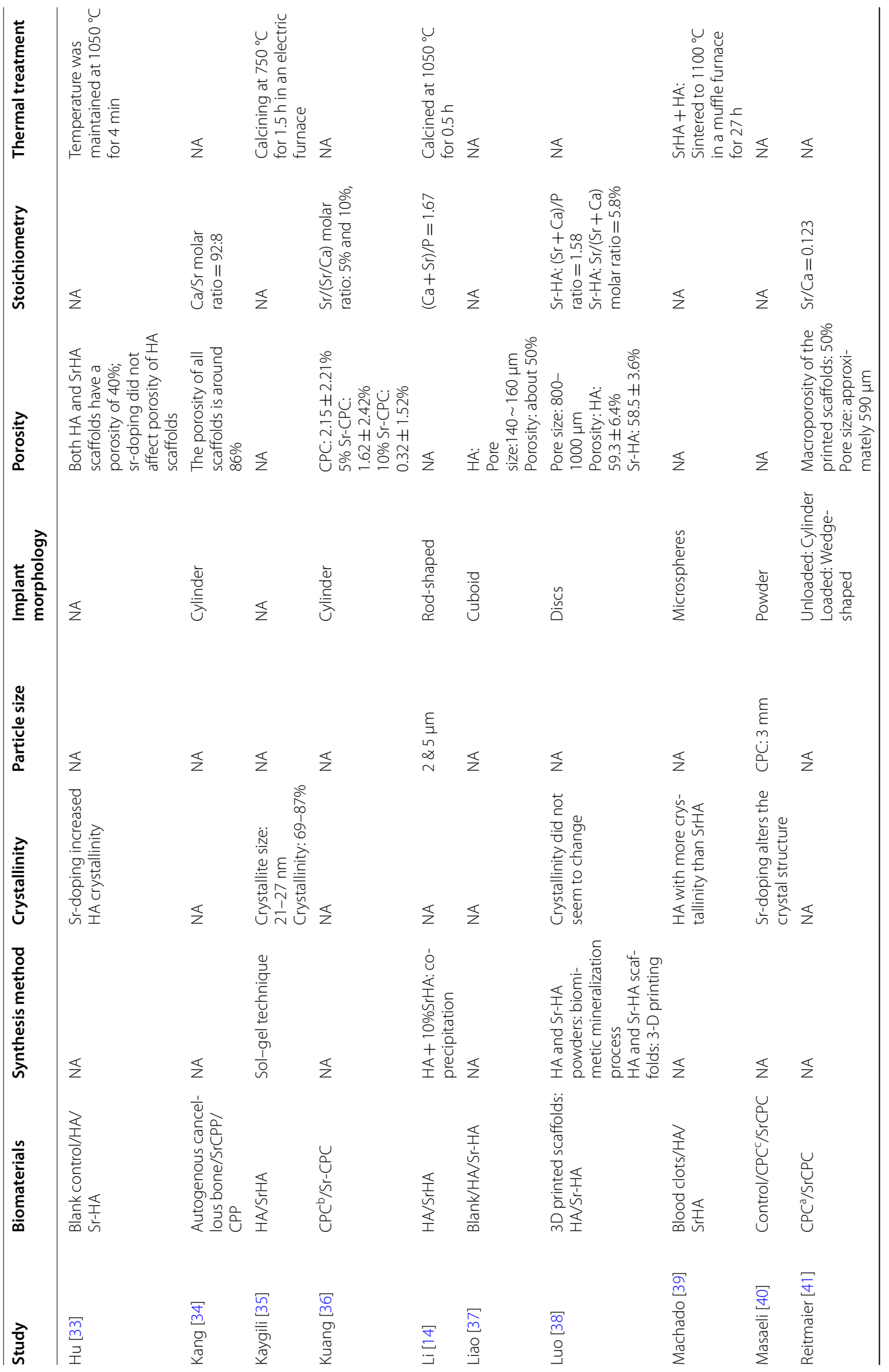




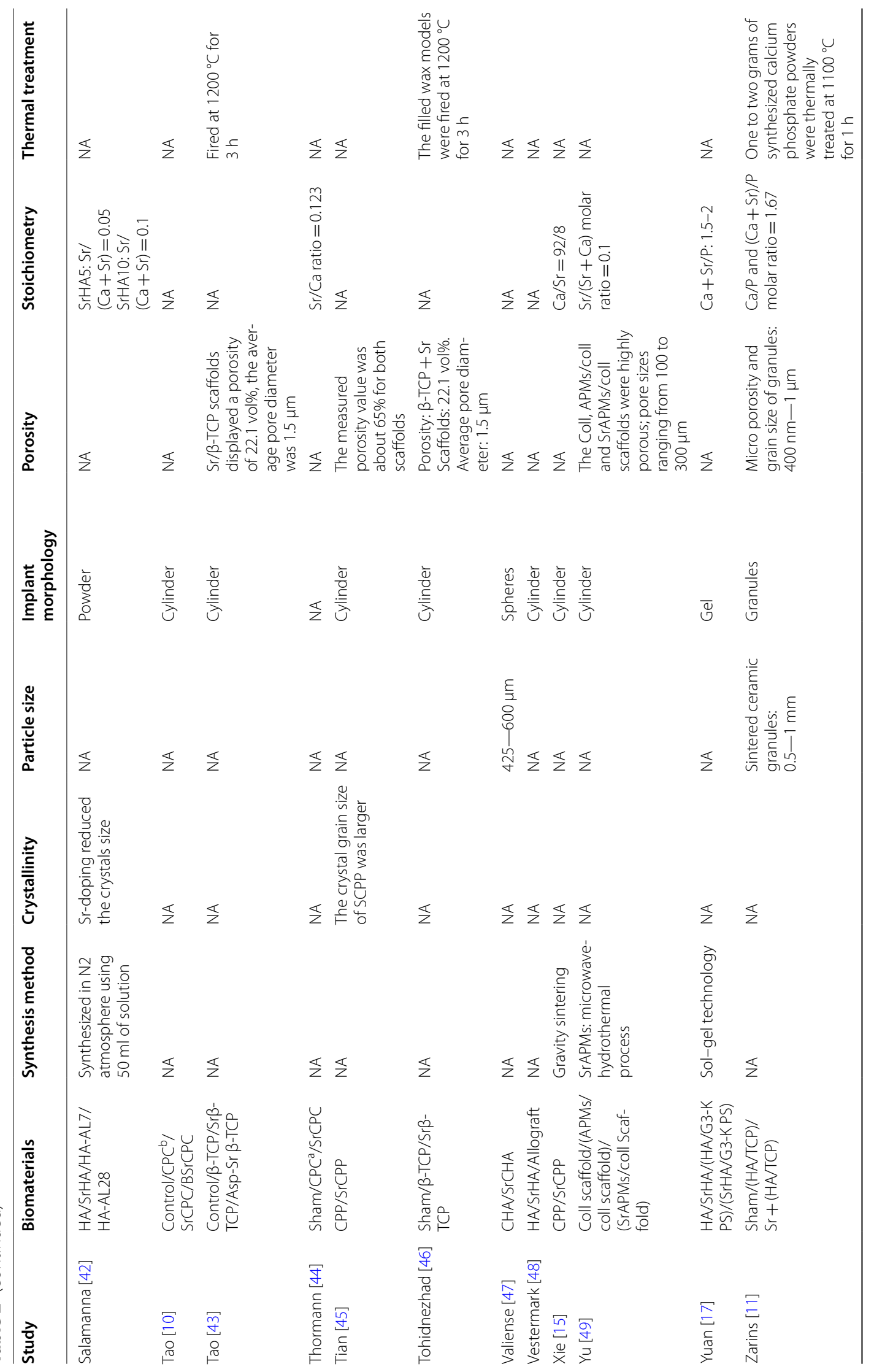




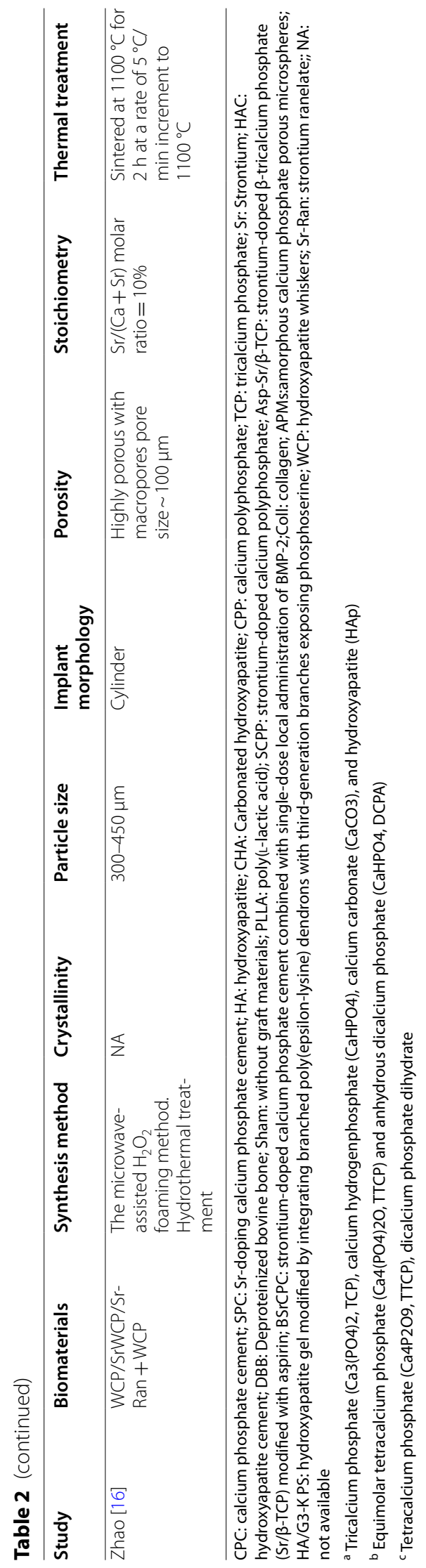




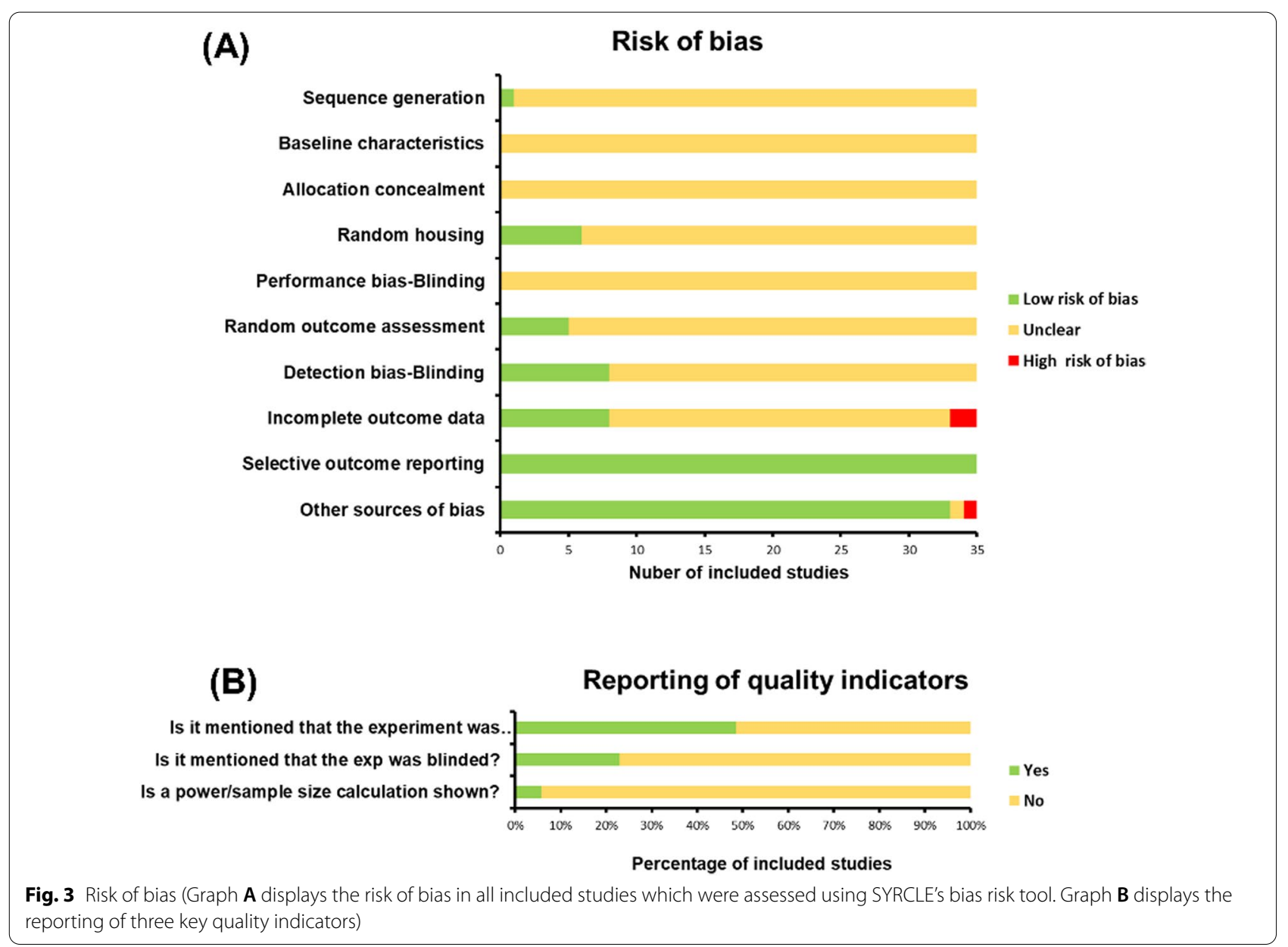

showed that the absorption of Sr-doped HA materials was slower than that of $\mathrm{Sr}$-free materials, albeit with no statistical significance. For the other three types of materials, the absorption of $\mathrm{Sr}$-doped materials was faster than that of the control group. The differences between $\beta$-TCP and CPC were statistically significant (Fig. 7).

\section{Publication bias}

Funnel plots showed no significant publication bias, as no significant asymmetry was detected (Additional file 3: Fig. S5).

\section{Discussion}

Synthetic calcium phosphate bone substitutes have been widely used for bone defect regeneration. To overcome the limitations of calcium phosphate materials, researchers are continuously proposing new methods. In recent years, many researchers have focused on adding inorganic ion Sr to calcium phosphate materials to improve their in vivo performance. However, at present, there is no consensus on whether $\mathrm{Sr}$ supplementation can significantly promote the biological and in vivo efficacy of bone replacement materials, to the best of our knowledge. Therefore, this paper systematically reviewed relevant in vivo studies and conducted a quantitative meta-analysis. The results showed that the $\mathrm{Sr}$-enhanced material significantly promoted the formation of new bone in the bone defect area, and the material was more easily absorbed. This is similar to the results of a previous study [50].

\section{Bone formation}

The specific mechanism by which Sr-containing materials promote osteogenesis is still unclear. Bone morphogenetic protein-2 (BMP-2)/Smad-1 and the osteoprotegerin (OPG)/receptor activator of the nuclear factor- $\mathrm{kB}$ ligand (RANKL) are two important signalling pathways for regulating osteogenesis. Previous studies have shown that bone remodelling regulates osteoblasts and osteoclasts through the BMP-2/Smad1 and OPG/RANKL signalling pathways, and is capable of bi-directional signalling [51, 52]. $\mathrm{Sr}$ is believed to have both osteogenic (anabolic) and antiabsorptive (catabolic) effects [36, 53]. Many studies have shown that the addition of $\mathrm{Sr}$ could stimulate the 


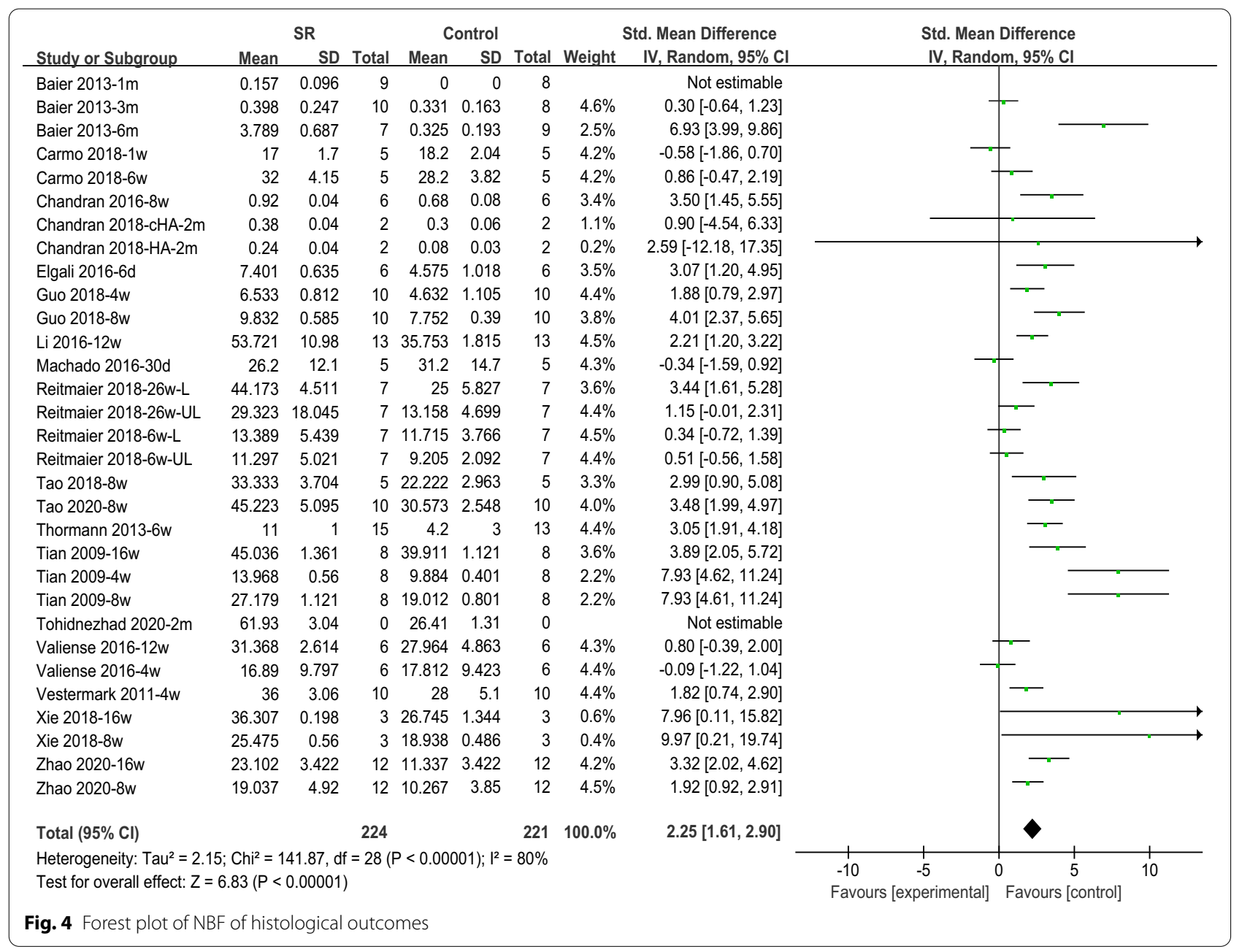

differentiation of MSCs or other osteoblast lineages [54, 55]. The expression of osteoblast markers (alkaline phosphatase $[\mathrm{ALP}]$, bone sialoprotein, and osteocalcin) was increased to promote the formation of bone nodules [53, $56]$, while reducing the differentiation and proliferation of osteoclasts [57].

Osteoporosis is a systemic bone disease characterized by bone loss and structural destruction. Owing to osteoblastic degeneration, increased osteoclast function, and insufficient bone formation ability, the treatment of bone defects in patients with osteoporosis is very challenging [1] In this study, a meta-analysis of nine studies [10, $14,16,22,25,26,32,43,44]$ using osteoporosis models was conducted, and the results showed that the addition of $\mathrm{Sr}$ could significantly promote NBF in animals with osteoporosis.

Sr has been shown to promote NBF by activating CA-sensitive receptors and inhibit bone resorption by blocking the expression of receptor activators of the nuclear factor $\mathrm{\kappa B}$ ligand (RANKL) [58, 59]. Animal studies on Sr-doped materials have shown that the enhancement of bone formation could be related to the release of $\mathrm{Sr}$ ions during the degradation process [45]. Biomaterials containing $\mathrm{Sr}$ exhibit high expression of physiologically active signalling molecules, such as OPG, NFkB 105, ALP, Col-1 $\alpha$, osteocalcin, osteopontin, and BMP 2/4 [57, 60-63]. This means that Sr-rich materials stimulated the release of these molecules more than calcium phosphate alone or simply the trauma itself.

In addition, the $\mathrm{Sr}$ released by bioceramics has been shown to stimulate angiogenesis by increasing the secretion of the cytokines that promote cell angiogenesis [64, 65]. A previous study has shown that, one week after SrWCP implantation in osteoporotic animals, vascularlike structures were formed in the pores in the central region of the bioceramics [16]. This angiogenesis is necessary for bone regeneration because these new blood vessels supply the oxygen, nutrients, and cells required for bone formation. 
Table 3 Subgroup analysis of the included papers for outcome new bone formation (NBF; SMD)

\begin{tabular}{|c|c|c|c|c|}
\hline Subgroup & Number of comparisons & Number of defects & Effect estimate SMD [95\% Cl] & Heterogeneity $\left(I^{2}\right)$ \\
\hline \multicolumn{5}{|l|}{ Disease } \\
\hline Health & 17 & 202 & $1.85[0.95,2.76]$ & $81 \%$ \\
\hline Osteoporosis & 14 & 243 & $2.73[1.94,3.52]$ & $71 \%$ \\
\hline \multicolumn{5}{|l|}{ Animal } \\
\hline Rat & 15 & 267 & $2.42[1.62,3.22]$ & $79 \%$ \\
\hline Rabbit & 7 & 84 & $4.32[1.78,6.86]$ & $88 \%$ \\
\hline Sheep & 7 & 74 & $0.85[-0.03,1.72]$ & $52 \%$ \\
\hline \multicolumn{5}{|l|}{ Material } \\
\hline $\mathrm{HA}$ & 7 & 120 & $2.18[1.19,3.17]$ & $74 \%$ \\
\hline$\beta-T C P$ & 2 & 20 & $3.48[1.99,4.97]$ & Not applicable \\
\hline CPC & 9 & 145 & $1.98[0.85,3.12]$ & $83 \%$ \\
\hline CPP & 5 & 60 & $6.60[4.09,9.12]$ & $52 \%$ \\
\hline \multicolumn{5}{|l|}{ Period } \\
\hline $1 \mathrm{~m}$ & 6 & 95 & $1.68[0.08,3.29]$ & $86 \%$ \\
\hline $2 \mathrm{~m}$ & 10 & 116 & $3.52[2.35,4.69]$ & $55 \%$ \\
\hline $3 \mathrm{~m}$ & 2 & 44 & $1.10[-0.08,2.29]$ & $74 \%$ \\
\hline $4 \mathrm{~m}$ & 3 & 46 & $3.59[2.54,4.64]$ & $0 \%$ \\
\hline
\end{tabular}

HA: hydroxyapatite; $\beta$-TCP: beta-tricalcium phosphate; CPC: Calcium phosphate cements; CPP: Calcium polyphosphate; m: month

\begin{tabular}{|c|c|c|c|c|c|c|c|c|c|c|c|}
\hline \multirow[b]{2}{*}{ Study or Subgroup } & \multicolumn{3}{|c|}{ SR } & \multicolumn{2}{|c|}{ Control } & \multirow[b]{2}{*}{ Total } & \multirow{2}{*}{ Weight } & \multirow{2}{*}{$\begin{array}{l}\text { Std. Mean Difference } \\
\text { IV, Random, } 95 \% \mathrm{Cl}\end{array}$} & \multirow{2}{*}{\multicolumn{3}{|c|}{$\begin{array}{l}\text { Std. Mean Difference } \\
\text { IV, Random, } 95 \% \mathrm{Cl}\end{array}$}} \\
\hline & Mean & SD & Total & Mean & $\mathrm{SD}$ & & & & & & \\
\hline Guo 2018-4w & 49.871 & 3.556 & 10 & 48.934 & 0.842 & 10 & $13.8 \%$ & $0.35[-0.54,1.23]$ & & & \\
\hline Guo 2018-8w & 58.573 & 4.866 & 10 & 51.93 & 1.871 & 10 & $12.8 \%$ & $1.73[0.67,2.79]$ & & & \\
\hline Tao 2018-8w & 0.4 & 0.084 & 5 & 0.345 & 0.084 & 5 & $11.5 \%$ & $0.59[-0.69,1.87]$ & & & \\
\hline Tao 2020-8w & 37.333 & 3.556 & 10 & 26.222 & 2.667 & 10 & $10.5 \%$ & $3.39[1.92,4.85]$ & & & \\
\hline Yu 2017-8w & 48.3 & 11.75 & 12 & 20.64 & 7.33 & 12 & $12.2 \%$ & $2.73[1.56,3.89]$ & & & \\
\hline Yuan 2018-8w & 18.491 & 3.567 & 6 & 17.558 & 3.786 & 6 & $12.3 \%$ & $0.23[-0.90,1.37]$ & & & \\
\hline Zhao 2020-16w & 26.457 & 1.889 & 12 & 21.889 & 2.205 & 12 & $12.9 \%$ & $2.15[1.11,3.19]$ & & & \\
\hline Zhao 2020-8w & 21.417 & 2.205 & 12 & 20.315 & 0.945 & 12 & $14.1 \%$ & $0.63[-0.20,1.45]$ & & & \\
\hline Total $(95 \% \mathrm{Cl})$ & & & 77 & & & 77 & $100.0 \%$ & $1.42[0.65,2.18]$ & & & \\
\hline $\begin{array}{l}\text { Heterogeneity: } \mathrm{Tau}^{2}= \\
\text { Test for overall effect: }\end{array}$ & $\begin{array}{l}0.90 ; \mathrm{Chi}^{2} \\
\mathrm{Z}=3.63(\end{array}$ & $\begin{array}{l}2=28.36 \\
P=0.0\end{array}$ & $\begin{array}{l}0, d f= \\
003)\end{array}$ & $7(P=0.0$ & $0002) ; 1^{2}$ & $=75 \%$ & & & $\begin{array}{ccc}-4 & -2 & 0 \\
\text { Favours [experimental] }\end{array}$ & $\begin{array}{c}2 \\
\text { Favours [control] }\end{array}$ & 4 \\
\hline
\end{tabular}

In addition, different animal models, implant sites, and bone defect sizes may also influence the conditions of NBF and material degradation. It is generally believed that experimental research on large animals reflects clinical practice more closely; however, there are few studies using large animals. Only three studies on sheep have been included in this meta-analysis on NBF, while no meta-analysis on remaining material could be conducted owing to the limited number of studies on large animals. The subgroup analysis of different animal types showed that Sr-doping significantly promoted the formation of new bone in sheep, dogs, rabbits, and rat. However, it should be noted that, although subgroup analyses were conducted, the results of these meta-analyses still exhibit significant heterogeneity among studies. This could be related to differences in implant sites (calvaria, femur, radius, etc.), bone defect sizes $(3 \mathrm{~mm}, 5 \mathrm{~mm}, 10 \mathrm{~mm}$, etc.), sample size, and experimental design.

\section{Material degradation}

Histological assessments were used to quantitatively determine the residual materials by conducting a metaanalysis. The percentages of remaining materials according to different implantation periods are shown in Fig. 6. At less than 1 month, the degradation rate of Sr-doped materials was lower than that of the control group. However, the degradation rate of the Sr-doped group was significantly higher at longer periods (greater than 


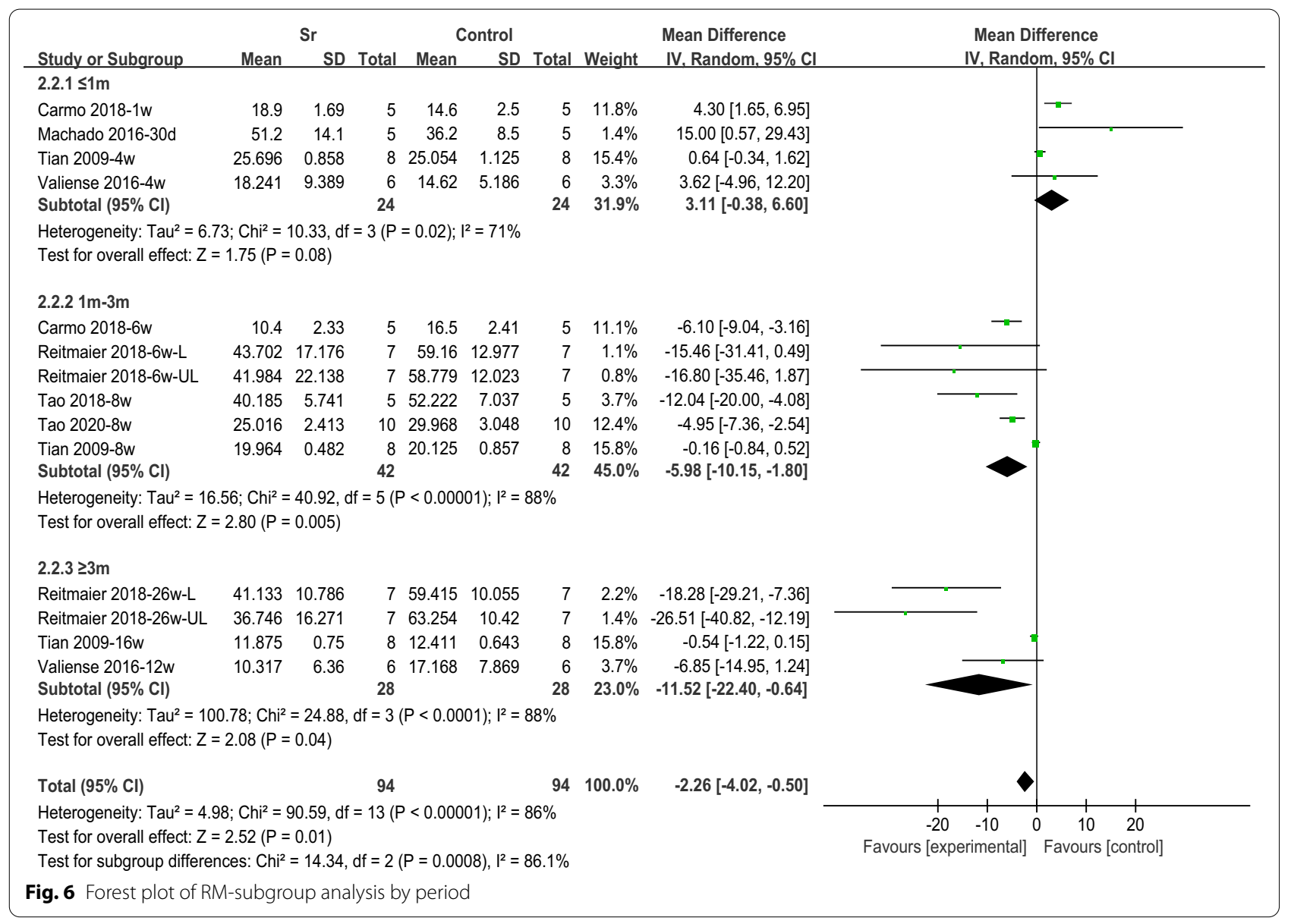

1 month). This indicates that the degradation rate of Srdoped materials may gradually increase with time, and is significantly higher than that of the Sr-free group. Studies showed that different types of calcium phosphate would affect the degradation rate of materials. It is generally believed that HA is more difficult to degrade. In the subgroup analysis for different material types, it can be seen that the residual rate of Sr-doped materials in the HA group is higher than that in the control group. However, the three studies $[23,39,47]$ in the HA group with high material residual rates all had shorter observation periods $(1 \mathrm{w}, 4 \mathrm{w}$, and $30 \mathrm{~d})$. Therefore, this may suggest that the doping of $\mathrm{Sr}$ has a time-dependent effect on the material absorption.

Although enhanced degradations of Sr-doped materials were reported in studies in vitro and in vivo, the underlying mechanism remains unclear and requires further investigation. Some researchers believe that the degradation rate of CPP scaffolds in vivo is usually affected by the initial size of the particles during scaffold formation, the scaffold structure, the implantation site, and other factors [66]. The doping of Sr was generally carried out through ion substitution, where $\mathrm{Sr}^{2+}$ could replace $\mathrm{Ca}^{2+}$ ions. Previous studies have shown that the ion radii of bioinorganic ions usually differ from those of substituted ions, and their supplementation could change the crystallinity, lattice parameters, crystal size, morphology, stability, biological activity, bone conductivity, and solubility of the material $[6,13,40]$. These physical and chemical changes may alter the fragmentation and biological absorption of biomaterials [13, 47]. According to Chandran et al. [25] and Landi et al. [67], the increased dissolution rates could be a result of the combined action of the increased pore size and the amorphous properties of SrHA particles.

In our opinion, the faster degradation rates of Sr-doped materials could also contribute to the improved release of bioinorganic substances and, thus, accelerate NBF.

\section{Implications and limitations}

Our study is likely to be the first report that systematically reviews relevant studies on Sr-doped (CaP)-based materials and conducts sub-group meta-analyses according to different influence factors. Furthermore, our study revealed the effect of $\mathrm{Sr}$-enhanced materials in vivo, 


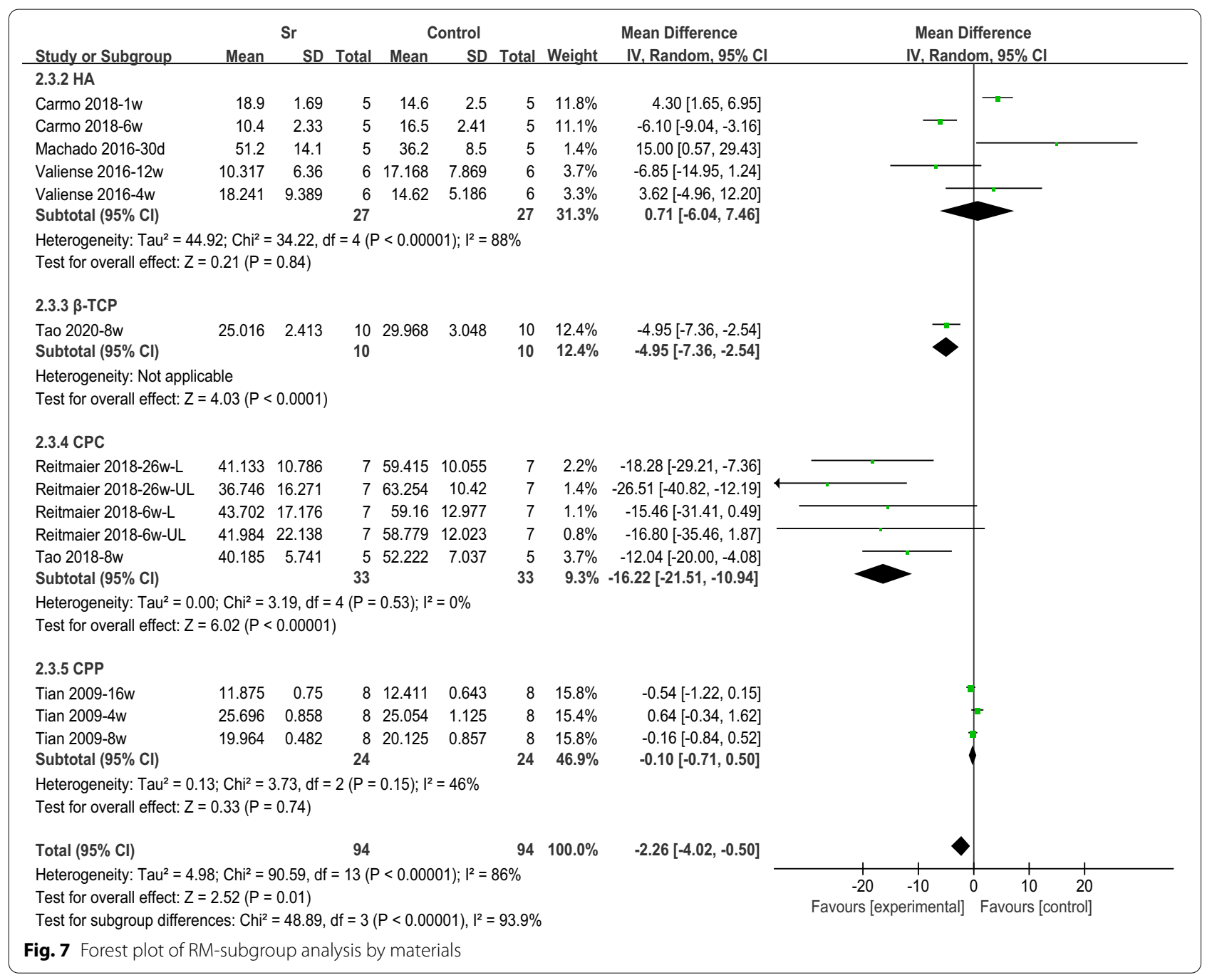

which provides a good basis for their further research and clinical application.

However, our study also has certain limitations. First, in this study, high heterogeneity was found in the meta-analysis of NBF and residual materials. Subgroup analyses based on material type, implantation period, experimental animal species, etc., also had high heterogeneity. In view of the significant heterogeneity among the studies included in our meta-analyses, caution should be exercised when generalizing our conclusions. It is suggested that homogenized study settings should be adopted in subsequent studies to provide more convincing evidence for clinical applications. Second, the quality of the included studies is not high enough. The details of sample size estimation and randomization methodology were not found in most studies. Finally, although $\mathrm{Sr}$ has a beneficial effect on bone formation, its potential negative effects should also be taken into account, especially in high doses $[29,68,69]$. A dose-dependent effect of $\mathrm{Sr}$ on osteoblasts could be detected in some in vitro studies [70]. Animal studies have shown that the Sr dosage was very important, as high doses could cause osteomalacia [71]. In this study, the included studies used different concentrations of $\mathrm{Sr}$, and some did not report relevant data. Therefore, it is necessary to further explore the optimal concentration of $\mathrm{Sr}$.

\section{Relevant studies during 2021}

During the past year (2021), another four in vivo studies relevant to this topic were found. One of them focused on strontium-doped nano hydroxyapatite-gelatin ( $\mathrm{Sr}$ $\mathrm{nHA}-\mathrm{Gel})$. An in vitro study and the in vivo repair of critical-sized cranial defects confirmed that Sr-nHA gel had relatively effective bone regeneration ability [72]. Another article focused on strontium-releasing nanoscale cement. In vivo and in vitro experiments showed that SR nano bone cement had the dual effects of osteoclast inhibition and osteogenic stimulation, indicating good potential for 
the treatment of osteoporotic bone defects [73]. The effect of the scaffold degradation rate on osteogenesis has been widely researched. Miao et al. [74] prepared strontiumdoped calcium sulfate $(\mathrm{SrCSH})$ and strontium-doped tricalcium phosphate microsphere (Sr-TCP) scaffolds. In the experiment on repairing osteoporotic femoral defects, they found that, when the degradation rate of the scaffold matched the growth rate of new bone, the rapid repair of osteoporotic bone defects was promoted. In contrast, the slow degradation of scaffold materials hindered the growth of new bone to a certain extent. This study further clarified the importance of the scaffold degradation rate in the repair of osteoporotic bone defects. Vascularized bone tissue engineering is of great significance for the reconstruction of critical bone defects. The application of calcium phosphate cement in vascularized bone tissue engineering is limited due to the lack of consequent angiogenesis and unsatisfactory physical and chemical properties. Wu et al. [75] developed a strontium-reinforced calcium phosphate composite cement based on the reported osteogenic and angiogenic properties of CPHCstar and BaSO4-incorporated calcium phosphate hybrid cement; further, Sr ions could improve the biological and physicochemical properties of CPC. In vivo and in vitro studies have shown that the material has the dual potential of osteogenesis and angiogenesis.

The aforementioned studies exhibited the significance of strontium-doped bone substitute materials in promoting bone regeneration, and also formed the basis for research into bone substitute materials.

\section{Conclusion}

According to the results of the systematic review and meta-analyses herein, $\mathrm{Sr}$ supplementation is advantageous in terms of promoting NBF and accelerating material degradation. The type of material (HA, $\beta$-TCP, CPC, or CPP) does not seem to affect NBF. In terms of material degradation, HA seems to degrade slowly, while the other three categories degraded more rapidly. However, the existing meta-analysis results all suggested high heterogeneity and no statistical significance. Therefore, further research is required to verify the differences between materials and further verify the conclusions of this study. Determining the optimum concentrations of $\mathrm{Sr}$ and the best $\mathrm{Sr}$-doped calcium phosphate materials is an important future research direction. In addition, the angiogenic potential of materials could be another research direction worth focusing on, in addition to osteogenesis.

\section{Abbreviations}

Sr: Strontium; NBF: New bone formation; RM: Remaining material; BV/TV: Bone volume/tissue volume; SYRCLE: Systematic Review Centre for Laboratory Animal Experimentation; RoB: Risk of Bias; 95\% Cl: 95\% Confidence interval; SMD: Standardized mean differences; MD: Mean differences; CPC: Calcium phosphate cement; SPC: Sr-doping calcium phosphate cement; HA: Hydroxyapatite; TCP: Tricalcium phosphate; TTCP: Anhydrous dicalcium phosphate; DCPA: Tetracalcium phosphate; CHA: Carbonated hydroxyapatite; CPP: Calcium polyphosphate; HAC: Hydroxyapatite cement; DBB: Deproteinized bovine bone; PLLA: Poly(L-lactic acid); SCPP: Strontium-doped calcium polyphosphate; Asp-Sr/ $\beta$-TCP: Strontium-doped $\beta$-tricalcium phosphate (Sr/ $\beta$-TCP) modified with aspirin; BSrCPC: Strontium-doped calcium phosphate cement combined with single-dose local administration of BMP-2; Coll: Collagen; APMs: Amorphous calcium phosphate porous microspheres; HA/G3-K PS: Hydroxyapatite gel modified by integrating branched poly(epsilon-lysine) dendrons with third-generation branches exposing phosphoserine; WCP: Hydroxyapatite whiskers; Sr-Ran: Strontium ranelate; NA: Not available; ALP: Alkaline phosphatase; BMP-2: Bone morphogenetic protein-2; OPG: Osteoprotegerin; RANKL: Receptor activator of the nuclear factor-kB ligand; Sr-nHA-Gel: Strontium-doped nano hydroxyapatite-gelatin.

\section{Supplementary Information}

The online version contains supplementary material available at https://doi. org/10.1186/s12903-022-02092-7.

Additional file 1: Table S1. Literature search-strategy for PubMed.

Additional file 2: Table S2. Literature search-strategy for Embase (via OVIDSP).

Additional file 3: Supplementary figures.

Acknowledgements

Not applicable.

Authors' contributions

JC designed the study. MY, YO, YL and RL performed the search, study selection, data curation, statistical analysis and wrote the manuscript. YF, WW, LZ, and $X Y$ participated in writing the article manuscript. All authors read and approved the final manuscript.

\section{Funding}

Funding was provided by the Fujian Province Natural Science Foundation of China (No. 2018J01818), Youth Scientific Research Project of Fujian Provincial Commission of Health and Family Planning (No. 2017-2-34) and Educational Research Project for Young and Middle-aged Teachers of Fujian Provincial Department of Education (NO. JAT200150).

Availability of data and materials

Data analysed during this study was included in this article and its supplementary information files.

\section{Declarations}

Ethics approval and consent to participate Not applicable.

Consent for publication

Not applicable.

Competing interests

The authors have declared that no competing interests exists.

\section{Author details}

${ }^{1}$ Fujian Key Laboratory of Oral Diseases and Fujian Provincial Engineering Research Center of Oral Biomaterial and Stomatological Key Laboratory of Fujian College and University, School and Hospital of Stomatology, Fujian Medical University, Fuzhou 350002, China. ${ }^{2}$ Department of Oral Implantology, Affiliated Stomatological Hospital of Fujian Medical University, Fuzhou 350002, China. ${ }^{3}$ ORAL Center, Fujian Provincial Governmental Hospital (Affiliated Hospital of Fujian Health College), Fuzhou 350003, China. ${ }^{4}$ Fujian Provincial Key Laboratory of Polymer Materials, College of Chemistry and Materials Science, 
Fujian Normal University, Fuzhou 350007, China. ${ }^{5}$ Institute of Stomatology and Research Center of Dental and Craniofacial Implants, School and Hospital of Stomatology, Fujian Medical University, Fuzhou, China.

Received: 23 July 2021 Accepted: 21 February 2022 Published online: 08 March 2022

\section{References}

1. Ball AN, Donahue SW, Wojda SJ, Mcllwraith CW, Kawcak CE, Ehrhart N, et al. The challenges of promoting osteogenesis in segmental bone defects and osteoporosis. J Orthop Res. 2018;36:1559-72.

2. Jiao $H$, Xiao E, Graves DT. Diabetes and its effect on bone and fracture healing. Curr Osteoporos Rep. 2015;13:327-35.

3. Dimitriou R, Mataliotakis Gl, Angoules AG, Kanakaris NK, Giannoudis PV. Complications following autologous bone graft harvesting from the iliac crest and using the RIA: a systematic review. Injury. 2011;42(Suppl 2):S3-15.

4. Liang F, Leland H, Jedrzejewski B, Auslander A, Maniskas S, Swanson $J$, et al. Alternatives to autologous bone graft in alveolar cleft reconstruction: the state of alveolar tissue engineering. J Craniofac Surg. 2018;29:584-93.

5. Jasser RA, AlSubaie A, AlShehri F. Effectiveness of beta-tricalcium phosphate in comparison with other materials in treating periodontal infra-bony defects around natural teeth: a systematic review and metaanalysis. BMC Oral Health. 2021;21:219.

6. Habibovic P, Barralet JE. Bioinorganics and biomaterials: bone repair. Acta Biomater. 2011;7:3013-26.

7. Wan B, Wang R, Sun Y, Cao J, Wang H, Guo J, et al. Building osteogenic microenvironments with strontium-substituted calcium phosphate ceramics. Front Bioeng Biotechnol. 2020;8:591467.

8. Mohan BG, Shenoy SJ, Babu SS, Varma HK, John A. Strontium calcium phosphate for the repair of leporine (Oryctolagus cuniculus) ulna segmental defect. J Biomed Mater Res A. 2013;101:261-71.

9. Deng C, Zhu H, Li J, Feng C, Yao Q, Wang L, et al. Bioactive scaffolds for regeneration of cartilage and subchondral bone interface. Theranostics. 2018;8:1940-55.

10. Tao Z, Zhou W, Jiang $Y, W u X, X u Z$, Yang $M$, et al. Effects of strontiummodified calcium phosphate cement combined with bone morphogenetic protein-2 on osteoporotic bone defects healing in rats. J Biomater Appl. 2018;33:3-10.

11. Zarins J, Pilmane M, Sidhoma E, Salma I, Locs J. Immunohistochemical evaluation after Sr-enriched biphasic ceramic implantation in rabbits femoral neck: comparison of seven different bone conditions. J Mater Sci Mater Med. 2018:29:119.

12. Ehret C, Aid-Launais R, Sagardoy T, Siadous R, Bareille R, Rey S, et al. Strontium-doped hydroxyapatite polysaccharide materials effect on ectopic bone formation. PLOS ONE. 2017;12:e184663.

13. Ge M, Ge K, Gao F, Yan W, Liu H, Xue L, et al. Biomimetic mineralized strontium-doped hydroxyapatite on porous poly(L-lactic acid) scaffolds for bone defect repair. Int J Nanomed. 2018;13:1707-21.

14. Li Y, Shui $X$, Zhang L, Hu J. Cancellous bone healing around strontiumdoped hydroxyapatite in osteoporotic rats previously treated with zoledronic acid. J Biomed Mater Res B Appl Biomater. 2016;104:476-81.

15. Xie H, Gu Z, He Y, Xu J, Xu C, Li L, et al. Microenvironment construction of strontium-calcium-based biomaterials for bone tissue regeneration: the equilibrium effect of calcium to strontium. J Mater Chem B. 2018;6:2332-9.

16. Zhao R, Chen S, Zhao W, Yang L, Yuan B, loan VS, et al. A bioceramic scaffold composed of strontium-doped three-dimensional hydroxyapatite whiskers for enhanced bone regeneration in osteoporotic defects. Theranostics. 2020;10:1572-89.

17. Yuan B, Raucci MG, Fan Y, Zhu X, Yang X, Zhang X, et al. Injectable strontium-doped hydroxyapatite integrated with phosphoserine-tethered poly(epsilon-lysine) dendrons for osteoporotic bone defect repair. J Mater Chem B. 2018;6:7974-84.

18. Hooijmans CR, Rovers MM, de Vries RB, Leenaars M, Ritskes-Hoitinga M, Langendam MW. SYRCLE's risk of bias tool for animal studies. BMC Med Res Methodol. 2014;14:43.
19. Hooijmans CR, Tillema A, Leenaars M, Ritskes-Hoitinga M. Enhancing search efficiency by means of a search filter for finding all studies on animal experimentation in PubMed. Lab Anim. 2010;44:170-5.

20. de Vries RB, Hooijmans CR, Tillema A, Leenaars M, Ritskes-Hoitinga M. Updated version of the Embase search filter for animal studies. Lab Anim. 2014;48:88.

21. Drevon D, Fursa SR, Malcolm AL. Intercoder reliability and validity of WebPlotDigitizer in extracting graphed data. Behav Modif. 2017:41:323-39.

22. Baier M, Staudt P, Klein R, Sommer U, Wenz R, Grafe I, et al. Strontium enhances osseointegration of calcium phosphate cement: a histomorphometric pilot study in ovariectomized rats. J Orthop Surg Res. 2013;8:16.

23. Carmo A, Sartoretto SC, Alves A, Granjeiro JM, Miguel FB, Calasans-Maia $J$, et al. Alveolar bone repair with strontium- containing nanostructured carbonated hydroxyapatite. J Appl Oral Sci. 2018;26:e20170084.

24. Cassino PC, Rosseti LS, Ayala OI, Martines MAU, Portugual LC, De Oliveira CG, et al. Potencial of different hydroxyapatites as biomaterials in the bone remodeling. Acta Cir Bras. 2018;33:816-23.

25. Chandran S, Babu SS, Vs HK, Varma HK, John A. Osteogenic efficacy of strontium hydroxyapatite micro-granules in osteoporotic rat model. J Biomater Appl. 2016:31:499-509.

26. Chandran S, Shenoy SJ, Babu SS, Nair PN, Varma HK, John A. Strontium hydroxyapatite scaffolds engineered with stem cells aid osteointegration and osteogenesis in osteoporotic sheep model. Colloids Surf B Biointerfaces. 2018;163:346-54.

27. Cheng C, Alt V, Pan L, Thormann U, Schnettler R, Strauss LG, et al. Preliminary evaluation of different biomaterials for defect healing in an experimental osteoporotic rat model with dynamic PET-CT (dPET-CT) using F-18-sodium fluoride (NaF). Injury. 2014;45:501-5.

28. Dagang $G$, Kewei $X$, Yong $H$. The influence of $\mathrm{Sr}$ doses on the in vitro biocompatibility and in vivo degradability of single-phase Sr-incorporated HAP cement. J Biomed Mater Res A. 2008;86:947-58.

29. Elgali I, Turri A, Xia W, Norlindh B, Johansson A, Dahlin C, et al. Guided bone regeneration using resorbable membrane and different bone substitutes: early histological and molecular events. Acta Biomater. 2016;29:409-23.

30. GuY, Liao D, Zhou Z. The experimental study of Sr-HAP on reconstructing mandibular bone defect. Zhonghua Kou Qiang Yi Xue Za Zhi. 2001;36:262-5.

31. Gu Z, Zhang X, Li L, Wang Q, Yu X, Feng T. Acceleration of segmental bone regeneration in a rabbit model by strontium-doped calcium polyphosphate scaffold through stimulating VEGF and bFGF secretion from osteoblasts. Mater Sci Eng C Mater Biol Appl. 2013;33:274-81.

32. Guo X, Wei S, Lu M, Shao Z, Lu J, Xia L, et al. RNA-Seq investigation and in vivo study the effect of strontium ranelate on ovariectomized rat via the involvement of ROCK1. Artif Cells Nanomed Biotechnol. 2018;46:629-41.

33. Hu B, Meng ZD, Zhang YQ, Ye LY, Wang CJ, Guo WC. Sr-HA scaffolds fabricated by SPS technology promote the repair of segmental bone defects. Tissue Cell. 2020;66:101386.

34. Kang P, Xie X, Tan Z, Yang J, Shen B, Zhou Z, et al. Repairing defect and preventing collapse of femoral head in a steroid-induced osteonecrotic of femoral head animal model using strontium-doped calcium polyphosphate combined BM-MNCs. J Mater Sci Mater Med. 2015;26:80.

35. Kaygili O, Keser S, Kom M, Eroksuz Y, Dorozhkin SV, Ates T, et al. Strontium substituted hydroxyapatites: Synthesis and determination of their structural properties, in vitro and in vivo performance. Mater Sci Eng C Mater Biol Appl. 2015;55:538-46.

36. Kuang GM, Yau WP, Wu J, Yeung KWK, Pan H, Lam WM, et al. Strontium exerts dual effects on calcium phosphate cement: Accelerating the degradation and enhancing the osteoconductivity both in vitro and in vivo. J Biomed Mater Res Part A. 2015;103:1613-21.

37. Liao D, Zhou Z, Gu Y, Chen D. A fundamental study on bioreactions of Sr-HA. Hua Xi Kou Qiang Yi Xue Za Zhi. 2002;20(172-4):183.

38. Luo Y, Chen S, Shi Y, Ma J. 3D printing of strontium-doped hydroxyapatite based composite scaffolds for repairing critical-sized rabbit calvarial defects. Biomed Mater. 2018;13:65004

39. Machado CP, Sartoretto SC, Alves AT, Lima IB, Rossi AM, Granjeiro JM, et al Histomorphometric evaluation of strontium-containing nanostructured hydroxyapatite as bone substitute in sheep. Braz Oral Res. 2016;30:e45. 
40. Masaeli R, Jafarzadeh KTS, Dinarvand R, Rakhshan V, Shahoon H, Hooshmand B, et al. Efficacy of the biomaterials 3wt\%-nanostrontiumhydroxyapatite-enhanced calcium phosphate cement (nanoSr-CPC) and nanoSr-CPC-incorporated simvastatin-loaded poly(lactic-co-glycolicacid) microspheres in osteogenesis improvement: An explorative multiphase experimental in vitro/vivo study. Mater Sci Eng C Mater Biol Appl. 2016:69:171-83

41. Reitmaier S, Kovtun A, Schuelke J, Kanter B, Lemm M, Hoess A, et al. Strontium(II) and mechanical loading additively augment bone formation in calcium phosphate scaffolds. J Orthop Res. 2018;36:106-17.

42. Salamanna F, Giavaresi G, Parrilli A, Torricelli P, Boanini E, Bigi A, et al. Antiresorptive properties of strontium substituted and alendronate functionalized hydroxyapatite nanocrystals in an ovariectomized rat spinal arthrodesis model. Mater Sci Eng C Mater Biol Appl. 2019;95:355-62.

43. Tao ZS, Zhou WS, Xu HG, Yang M. Aspirin modified strontium-doped beta-tricalcium phosphate can accelerate the healing of femoral metaphyseal defects in ovariectomized rats. Biomed Pharmacother. 2020;132:110911.

44. Thormann U, Ray S, Sommer U, ElKhassawna T, Rehling T, Hundgeburth $M$, et al. Bone formation induced by strontium modified calcium phosphate cement in critical-size metaphyseal fracture defects in ovariectomized rats. Biomaterials. 2013;34:8589-98.

45. Tian M, Chen F, Song W, Song Y, Chen Y, Wan C, et al. In vivo study of porous strontium-doped calcium polyphosphate scaffolds for bone substitute applications. J Mater Sci Mater Med. 2009;20:1505-12.

46. Tohidnezhad M, Kubo Y, Lichte P, Heigl T, Roch D, Barahmand PN, et al. Effects of strontium-doped $\beta$-tricalcium scaffold on longitudinal nuclear factor-kappa beta and vascular endothelial growth factor receptor-2 promoter activities during healing in a murine critical-size bone defect model. Int J Mol Sci. 2020;21:3208.

47. Valiense H, Barreto M, Resende RF, Alves AT, Rossi AM, Mavropoulos E, et al. In vitro and in vivo evaluation of strontium-containing nanostructured carbonated hydroxyapatite/sodium alginate for sinus lift in rabbits. J Biomed Mater Res B Appl Biomater. 2016;104:274-82.

48. Vestermark MT, Hauge EM, Soballe K, Bechtold JE, Jakobsen T, Baas J. Strontium doping of bone graft extender. Acta Orthop. 2011;82:614-21.

49. Yu W, Sun TW, Qi C, Ding Z, Zhao H, Chen F, et al. Strontium-doped amorphous calcium phosphate porous microspheres synthesized through a microwave-hydrothermal method using fructose 1,6-bisphosphate as an organic phosphorus source: application in drug delivery and enhanced bone regeneration. ACS Appl Mater Interfaces. 2017;9:3306-17.

50. Lodoso-Torrecilla I, Klein GR, Grosfeld EC, de Vries R, Habibovic P, Jansen $J A$, et al. Bioinorganic supplementation of calcium phosphate-based bone substitutes to improve in vivo performance: a systematic review and meta-analysis of animal studies. Biomater Sci. 2020;8:4792-809.

51. Ren C, Gong W, Li F, Xie M. Pilose antler aqueous extract promotes the proliferation and osteogenic differentiation of bone marrow mesenchymal stem cells by stimulating the BMP-2/Smad1, 5/Runx2 signaling pathway. Chin J Nat Med. 2019;17:756-67.

52. Ge YW, Feng K, Liu XL, Zhu ZA, Chen HF, Chang YY, et al. Quercetin inhibits macrophage polarization through the $p$-38alpha/beta signalling pathway and regulates OPG/RANKL balance in a mouse skull model. J Cell Mol Med. 2020;24:3203-16.

53. Bonnelye E, Chabadel A, Saltel F, Jurdic P. Dual effect of strontium ranelate: stimulation of osteoblast differentiation and inhibition of osteoclast formation and resorption in vitro. Bone. 2008;42:129-38.

54. Nardone V, Zonefrati R, Mavilia C, Romagnoli C, Ciuffi S, Fabbri S, et al. In vitro effects of strontium on proliferation and osteoinduction of human preadipocytes. Stem Cells Int. 2015;2015:87186.

55. Boanini E, Torricelli P, Fini M, Bigi A. Osteopenic bone cell response to strontium-substituted hydroxyapatite. J Mater Sci Mater Med. 2011;22:2079-88.

56. Zhu LL, Zaidi S, Peng Y, Zhou H, Moonga BS, Blesius A, et al. Induction of a program gene expression during osteoblast differentiation with strontium ranelate. Biochem Biophys Res Commun. 2007;355:307-11.

57. Geng Z, Wang X, Zhao J, Li Z, Ma L, Zhu S, et al. The synergistic effect of strontium-substituted hydroxyapatite and microRNA-21 on improving bone remodeling and osseointegration. Biomater Sci. 2018;6:2694-703.

58. Saidak Z, Marie PJ. Strontium signaling: molecular mechanisms and therapeutic implications in osteoporosis. Pharmacol Ther. 2012;136:216-26.
59. Tat SK, Pelletier JP, Mineau F, Caron J, Martel-Pelletier J. Strontium ranelate inhibits key factors affecting bone remodeling in human osteoarthritic subchondral bone osteoblasts. Bone. 2011;49:559-67.

60. Neves N, Linhares D, Costa G, Ribeiro CC, Barbosa MA. In vivo and clinical application of strontium-enriched biomaterials for bone regeneration: a systematic review. Bone Joint Res. 2017;6:366-75.

61. Andersen OZ, Offermanns $V$, Sillassen $M$, Almtoft KP, Andersen IH, Sorensen $\mathrm{S}$, et al. Accelerated bone ingrowth by local delivery of strontium from surface functionalized titanium implants. Biomaterials. 2013;34:5883-90

62. Jing D, Hao X, Xu F, Liu J, Xu F, Luo E, et al. Effects of local delivery of BMP2, zoledronate and their combination on bone microarchitecture, biomechanics and bone turnover in osteoporotic rabbits. Sci Rep. 2016;6:28537.

63. Lin K, Xia L, Li H, Jiang X, Pan H, Xu Y, et al. Enhanced osteoporotic bone regeneration by strontium-substituted calcium silicate bioactive ceramics. Biomaterials. 2013;34:10028-42.

64. Lin K, Liu P, Wei L, Zou Z, Zhang W, Qian Y, et al. Strontium substituted hydroxyapatite porous microspheres: surfactant-free hydrothermal synthesis, enhanced biological response and sustained drug release. Chem Eng J (Lausanne, Switzerland: 1996). 2013;222:49-59.

65. Mao L, Xia L, Chang J, Liu J, Jiang L, Wu C, et al. The synergistic effects of Sr and Si bioactive ions on osteogenesis, osteoclastogenesis and angiogenesis for osteoporotic bone regeneration. Acta Biomater. 2017;61:217-32.

66. Grynpas MD, Pilliar RM, Kandel RA, Renlund R, Filiaggi M, Dumitriu M. Porous calcium polyphosphate scaffolds for bone substitute applications in vivo studies. Biomaterials. 2002;23:2063-70.

67. Landi E, Uggeri J, Medri V, Guizzardi S. Sr, Mg cosubstituted HA porous macro-granules: potentialities as resorbable bone filler with antiosteoporotic functions. J Biomed Mater Res A. 2013;101:2481-90.

68. Lindahl C, Pujari-Palmer S, Hoess A, Ott M, Engqvist H, Xia W. The influence of Sr content in calcium phosphate coatings. Mater Sci Eng C Mater Biol Appl. 2015;53:322-30

69. Frasnelli M, Cristofaro F, Sglavo VM, Dire S, Callone E, Ceccato R, et al. Synthesis and characterization of strontium-substituted hydroxyapatite nanoparticles for bone regeneration. Mater Sci Eng C Mater Biol Appl. 2017;71:653-62.

70. Verberckmoes SC, De Broe ME, D'Haese PC. Dose-dependent effects of strontium on osteoblast function and mineralization. Kidney Int. 2003:64:534-43.

71. Fu DL, Jiang QH, He FM, Yang GL, Liu L. Fluorescence microscopic analysis of bone osseointegration of strontium-substituted hydroxyapatite implants. J Zhejiang Univ Sci B. 2012;13:364-71.

72. Song T, Zhao F, Wang Y, Li D, Lei N, Li X, Xiao Y, Zhang X. Constructing a biomimetic nanocomposite with the in situ deposition of spherical hydroxyapatite nanoparticles to induce bone regeneration. J Mater Chem B. 2021;9(10):2469-82.

73. Lee NH, Kang MS, Kim TH, Yoon DS, Mandakhbayar N, Jo SB, Kim HS, Knowles JC, Lee JH, Kim HW. Dual actions of osteoclastic-inhibition and osteogenic-stimulation through strontium-releasing bioactive nanoscale cement imply biomaterial-enabled osteoporosis therapy. Biomaterials. 2021;276:121025.

74. Miao Q, Jiang N, Yang Q, Hussein IM, Luo Z, Yang S, Yu B, Wang L. Multistage controllable degradation of strontium-doped calcium sulfate hemihydrate-Tricalcium phosphate microsphere composite as a substitute for osteoporotic bone defect repairing: Degradation behavior and bone response. Biomed Mater. 2021. https://doi.org/10.1088/1748-605X/ ac4323.

75. Wu X, Tang Z, Wu K, Bai Y, Lin X, Yang H, Yang Q, Wang Z, Ni X, Liu H, et al. Strontium-calcium phosphate hybrid cement with enhanced osteogenic and angiogenic properties for vascularised bone regeneration. J Mater Chem B. 2021;9(30):5982-97.

\section{Publisher's Note}

Springer Nature remains neutral with regard to jurisdictional claims in published maps and institutional affiliations. 